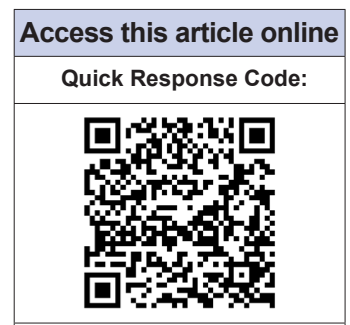

Website:

www.neurologyindia.com

DOI:

10.4103/0028-3886.236996

${ }^{1}$ Department of Surgical Sciences, Unit of Anesthesia and Intensive Care, University of

Torino, Torino, Italy, ${ }^{2}$ Neuroscience Center and JPN Apex Trauma

Center, All India

Institute of Medical

Sciences, New Delhi,

India

Address for

correspondence:

Dr. Anna Teresa Mazzeo,

Department of Surgical

Sciences, Unit of Anesthesia and Intensive Care, University of Torino,

Torino, Italy.

E-mail: anna.mazzeo@ unito.it

\title{
Intraoperative visual evoked potential monitoring for a safer endoscopic transsphenoidal surgery
}

\author{
Anna Teresa Mazzeo', Deepak Kumar Gupta ${ }^{2}$
}

The main goal of modern neurosurgery is removal of the lesion with preservation of neurological function. In order to reach this objective, intraoperative neurophysiological monitoring (IONM) has been introduced and evaluated with the aim to make the neurosurgeon aware of ongoing changes in neural function, allowing prompt corrective intervention to prevent postoperative deficits. The location of the lesion and the operative approach indicate which structures need to be monitored. Visual evoked potentials (VEPs) have been reported as a method of intraoperative monitoring of the visual pathways.

In this issue of the Neurology India, Nishimura et al., report their experience on VEP monitoring in endoscopic transnasal transsphenoidal surgery as a real-time visual function under total intravenous anesthesia (TIVA) ${ }^{[1]}$ The authors retrospectively studied the relationship between VEP waveform changes and postoperative visual function. Intraoperative flash VEP monitoring (16 red light emitting diodes [LEDs] placed bilaterally over closed eyes) was carried out during 82 endoscopic pituitary surgeries for 164 eyes. The authors concluded that intraoperative monitoring of VEP predicts postoperative visual function, and a reversible change in VEP indicates that visual function will be preserved. An amplitude that decreased $>50 \%$ from the control level was defined as a significant decrease. Among patients with decreased and restored VEP amplitude, $31 \%$ had improved postoperative visual function and $69 \%$ had unchanged postoperative visual function. In the group where VEP amplitude decreased and was maintained at a decline of $50 \%$ or less, all had unchanged visual function in the postoperative state. Authors used goggles that incorporated red LEDs capable of withstanding sterilization. Development of lighter and more durable

This is an open access journal, and articles are distributed under the terms of the Creative Commons Attribution-NonCommercial-ShareAlike 4.0 License, which allows others to remix, tweak, and build upon the work non-commercially, as long as appropriate credit is given and the new creations are licensed under the identical terms. goggles and a careful goggle setup resulted in a stable delivery of stimuli. They concluded that VEP can be steadily monitored in patients with corrected visual acuity $>0.1(6 / 60 \mathrm{~m})$; permanent VEP loss may indicate severe visual dysfunctions postoperatively while transient VEP changes do not indicate postoperative visual disturbance.

Visual pathway injuries can occur during surgery on anterior visual pathway lesions (pituitary tumors, craniopharyngiomas, chiasmal gliomas), pallidotomy procedures, ophthalmic aneurysm clipping, epilepsy surgery, and tumors near the visual cortex (occipital lobe gliomas). VEPs are time-locked electrophysiologic potentials recorded in response to standardized stimuli using scalp electrodes placed over the occipital lobes and visual cortical areas.

There are three main classes of VEPs based on the stimulus type used to generate the response: flash, pattern onset/offset, and pattern reversal. Of these, pattern reversal stimulation is the preferred stimulus in awake patient because it produces reliable and robust VEP waves. In intraoperative settings, it is impossible to perform pattern reversal VEP testing because the patient cannot voluntarily fixate on a distant object while under general anesthesia. For this reason, flash VEPs delivered to closed eyelids are used during the surgical procedures. The flash stimulus input to the retina is transmitted to the optic nerve, optic chiasm, optic tract, lateral geniculate body, optic radiation (geniculocalcarine tract), and visual cortical area, and the VEP waveform is recorded from the occipital region.

To obtain reproducible intraoperative flash VEP waveforms, the use of retinal flash stimulation devices utilizing high intensity LEDs, and a combination of electroretinography (to confirm that the flash stimulus has reached the retina) is required. In addition to VEPs, electroretinograms (ERGs)

How to cite this article: Mazzeo AT, Gupta DK. Intraoperative visual evoked potential monitoring for a safer endoscopic transsphenoidal surgery. Neurol India 2018;66:955-7. 
have been used to assess retinal function. ERGs can be recorded using flash (full-field flash ERG) or patterned stimuli (pattern ERG [PERG]). Light stimulating device consisting of 16 red high luminosity (20,000 lux) LEDs embedded in a soft round silicone disc to elicit VEP and recorded ERG permits flexibility of the device during surgical manipulation.

Intraoperative VEP is affected by various factors, viz, temperature (hypothermia attenuates VEP amplitude and latency gets extended, and waveforms disappear at 25-27 degree C), partial pressure of carbon dioxide in blood [hypocapnia causes changes in $\mathrm{pH}$ resulting in acceleration of conduction velocity of somatosensory evoked potential], hypoxia and hypotension (VEP amplitude is decreased and latency is extended), hematocrit values $<15 \%$, during turning of skin flap on forehead (may dislodge the LED stimulating electrode) and anesthetic agents. ${ }^{[2,3]}$ In some cases, VEPs change not as a result of damage to the optic pathway or ischemia but due to the anesthetics. As anesthetic agents suppress synaptic transmission, reactions from polysynaptic pathways (e.g., cortical recordings) are affected by anesthesia to a much greater extent than those recorded from oligosynaptic pathways. The optic pathway that is subjected to flash VEP monitoring is influenced by anesthetics because it passes through three synapses, including the lateral geniculate body, as it travels from the retina to the visual cortical area. Therefore, the neurosurgeon should discuss with the anesthesiologist what IONM modalities are planned and what anesthetic strategies are recommended for successful monitoring during the procedure. All inhaled anesthetics suppress flash VEPs by extending VEP latency and reducing their amplitude in a concentration-dependent manner, even at low concentrations. Nitrous oxide causes marked attenuation of the amplitude and the disappearance of waveforms when combined with a volatile anesthetic. Intravenous anesthetics generally affect evoked potentials less than inhaled anesthetics, and therefore, TIVA with propofol and opioids is the preferred anesthetic technique when IONM is planned..$^{[2,3]}$ Neuromuscular blocking drugs do not directly influence VEPs, but they should be avoided after intubation if concomitant mapping of motor function is planned. Among intravenous anesthetics, propofol has a small suppressive effect on flash VEPs, while thiopental extends the latency and attenuates the amplitude in a dose-dependent manner. The opioids, fentanyl and remifentanil, have no effects on flash VEPs in clinical doses.

The effective monitoring and application of VEPs is challenging and its value as an objective determination method is missing. Sato ${ }^{[3]}$ used a specific stimulus in 26 cases of brain surgery to overcome this limitation by identifying 2 groups of responses, that is, the response derived from the start of light emission (on response) and the response derived from the end of light emission (off response). Stable and recordable waves were observed by monitoring the off response, consisting of the P1-N1-P2 component, with a wave latency of approximately $100 \mathrm{msec}$. It was difficult to achieve stability by adjusting the light intensity and emission time using the on response. The off response was confirmed to be sufficiently stable for intraoperative monitoring. The off response was shown to have the capacity to function as a monitoring tool, providing more stable wave forms than the on response. Recording conditions could be adjusted to achieve a light-emitting time of $500 \mathrm{msec}$ and a light quantity of $8000 \mathrm{Lx} \cdot{ }^{[4]}$
Deficits like homonymous hemianopia from surgeries on intraaxial tumors in the occipital lobe or the temporal lobe handling in epilepsy surgeries can significantly impact the quality of life, leading to impairment in reading or driving vehicles. Gutzwiller et al. ${ }^{[4]}$ assessed the performance of intraoperative VEPs performed with white LEDs, with simultaneous recordings of ERGs. They used, as the alarm criterion, a VEP amplitude decrease of $>20 \%$, which allowed them to detect visual field changes larger than a discrete quadrantanopia, or a deterioration of an existing quadrantanopia. Use of white LEDs, instead of conventional red LEDs, can activate a larger area of the retina, i.e., all cones and rods, thereby entailing a larger activation of the visual pathways and the occipital cortex. One potential disadvantage of partial retinal stimulation covering only the macula is that the corresponding coverage of optic fibers along the optic nerve, tract, and radiations, as well as the occipital cortex is restricted anatomically, and therefore, damage to visual fibers that are projecting peripheral fields is underrecognized. The authors analyzed VEPs for 29 patients undergoing resection of a brain lesion. In $89.7 \%$ of the patients, steady VEPs and retinal responses were obtained for monitoring. The alarms correctly identified $66.7 \%$ of cases with any postoperative changes and $100 \%$ of cases with changes more severe than just a discrete quadrantanopia or deterioration of an existing quadrantanopia. ${ }^{[5]}$

Introduction of high-luminance devices with supramaximal stimulation have contributed to the success of VEP by improving the constant stimulus delivery. The two-step warning criterion is often used to assess VEP responses. First, a N75 amplitude decrease to less than $50 \%$ of the baseline (after excluding technical failures or anesthesia effect) warrants a temporary warning to be issued to the surgeon. In a second step, the succeeding VEP is used to either confirm or reject the warning. If the warning criterion is not reached, VEPs are then referred to as "stable". Transient VEP losses can also occur during bone drilling or if the patient throws generalized seizures intraoperatively. Interestingly, intraoperative VEP monitoring, which is limited to the duration of surgery, cannot document functional improvement. The combination of intraoperative VEP monitoring with ERG ascertains retinal light stimulation and thus improves the clinical utility of VEP. If VEPs are lost together with lost ERG, this indicates displacement of the LED and not damage of the visual pathways, which should be indicated by a combination of lost or diminished VEPs with preserved ERG. Real time monitoring is not yet possible as one needs more than 100 seconds to obtain one waveform. This introduces a delay between a possible damage of the visual pathway, VEP loss and the surgeon' s reaction. ${ }^{[6]}$

Marked methodologic improvement in VEP monitoring techniques was noted in a recently concluded meta-analysis on intraoperative monitoring of anterior visual pathways in 457 eyes. ${ }^{[5]}$ The predictive power for visual deterioration after surgery was approximately $60 \%$ and reached $100 \%$ when coupled with simultaneous monitoring of electroretinography [to avoid false negatives]. The sensitivity of visual evoked potentials for detection of deterioration was $47 \%$. Direct electrical stimulation of the distal optic nerve $(\mathrm{ON})$ and recording from proximal optic nerve can also be done for intraoperative evaluation of the integrity of the visual pathways. The vasculature of the 
ON has also been monitored using indocyanine green to avoid damaging the vascular supply of the ON. The best method to detect an improvement, although not in real time, is monitoring the decrease in FA (fractional anisotropy on intraoperative MRI) [100\% positive predictive value]. Combining VEP with FA may predict both improvement and deterioration. ${ }^{[7]}$

Electromagnetic navigation in endoscopic pituitary surgeries, when combined with continuous VEP monitoring, allows for the best available anatomic and real-time functional monitoring. The tracker-emitter distance needs to be optimized to avoid switching off navigation each time VEP is recorded. Kurozumi et al., in their series of 19 pituitary surgeries noted the optimal distance between the patient tracker and the emitter at $22.5 \pm 2.5 \mathrm{~cm}$ where the cumulative interference value was $0.7(<1.5)$. At less than $20 \mathrm{~cm}$, VEP monitoring had an intense noise and ERG was not detected. The advantage is that the signals are easy to monitor continuously. It is not necessary to switch off electromagnetic navigation. Optimizing the distance between the emitter and tracker minimizes VEP monitoring noise and allows for an accurate electromagnetic navigation. ${ }^{[8]}$

Intraoperative monitoring of the VEP under TIVA using flash LED goggles with simultaneous ERG is desirable for continuous monitoring of the visual function during surgeries that put the visual pathways at risk of injury wherein preserved VEP predicts preserved visual functions. It is now feasible to do endoscopic pituitary surgeries under electromagnetic navigation with intraoperative VEP recording for maximizing tumor resections and minimizing visual deficits. VEP monitoring using white instead of red LEDs can also detect new major visual field defects as a result of injuries to the posterior visual pathways.

Finally, even if IONM is a valuable adjunct to basal intraoperative monitoring in reducing complications and in improving outcomes, false-positive and false-negative errors can occur with monitoring. Therefore, IONM should be used within a knowledgeable surgical, neurophysiological and anesthesiological team, and can guide but never replace a neurosurgeon's judgment in preservation of neural structures. ${ }^{[9]}$

\section{References}

1. Nishimura F, Wajima D, Park YS, Motoyama Y, Nakagawa I, Yamada S, et al. Efficacy of the visual evoked potential monitoring in endoscopic transnasal transsphenoidal surgery as a real-time visual function. Neurol India 2018;66:1075-80.

2. Hayashi H, Kawaguchi M. Intraoperative monitoring of flash visual evoked potential under general anesthesia. Korean J Anesthesiol 2017;70:127-35.

3. Banoub M, Tetzlaff JE, Schubert A. Pharmacologic and physiologic influences affecting sensory evoked potentials. implications for perioperative monitoring. Anesthesiology 2003;99:716-37.

4. Sato A. Interpretation of the causes of instability of flash visual evoked potentials in intraoperative monitoring and proposal of a recording method for reliable functional monitoring of visual evoked potentials using a light-emitting device. J Neurosurg 2016;125:888-97.

5. Gutzwiller EM, Cabrilo I, Radovanovic I, Schaller K, Boëx C. Intraoperative monitoring with visual evoked potentials for brain surgeries. J Neurosurg 2018;30:1-7.

6. Luo Y, Regli L, Bozinov O, Sarnthein J. Clinical utility and limitations of intraoperative monitoring of visual evoked potentials. PLoS One. 2015;10:e0120525.

7. Metwali H, Kniese K, Fahlbusch R. Intraoperative monitoring of the integrity of the anterior visual pathways: A methodologic review and meta-analysis. World Neurosurg. 2018;110:217-25.

8. Kurozumi K, Kameda M, Ishida J, Date I. Simultaneous combination of electromagnetic navigation with visual evoked potential in endoscopic transsphenoidal surgery: Clinical experience and technical considerations. Acta Neurochir 2017;159:1043-8.

9. Skinner SA, Cohen BA, Morledge DE, McAuliffe JJ, Hastings JD, Yingling $\mathrm{CD}$, et al. Practice guidelines for the supervising professional: Intraoperative neurophysiological monitoring. J Clin Monit Comput 2014;28:103-11. 